A N N A L E S Annales de Bretagne et des Pays de l'Ouest

\title{
Conclusion
}

\section{La transition politique, observatoire original de la gendarmerie du xixe siècle}

Jean-Noël Luc

\section{(2) OpenEdition \\ Journals}

Édition électronique

URL : http://journals.openedition.org/abpo/87

DOI : $10.4000 /$ abpo.87

ISBN : 978-2-7535-1506-2

ISSN : 2108-6443

\section{Éditeur}

Presses universitaires de Rennes

\section{Édition imprimée}

Date de publication : 30 juin 2007

Pagination : 185-194

ISBN : 978-2-7535-0510-0

ISSN : 0399-0826

\section{Référence électronique}

Jean-Noël Luc, « La transition politique, observatoire original de la gendarmerie du XIXe siècle », Annales de Bretagne et des Pays de l'Ouest [En ligne], 114-2 | 2007, mis en ligne le 31 décembre 2009, consulté le 21 avril 2019. URL : http://journals.openedition.org/abpo/87 ; DOI : 10.4000/abpo.87 


\title{
Conclusion
}

\section{La transition politique, observatoire original de la gendarmerie du XIX ${ }^{\mathrm{e}}$ siècle}

\author{
Jean-Noël LuC \\ Professeur d'histoire contemporaine, université Paris IV-Sorbonne \\ Centre d'histoire du XIX siècle - Paris I-Paris IV
}

Rédigé sous la monarchie de Juillet, le témoignage de Jean-François La Roche, ancien lieutenant de la gendarmerie royale de Paris, illustre la résistance héroïque de son corps pendant l'insurrection des Trois Glorieuses :

"En voyant partir les autres troupes, mes hommes montèrent à cheval et me sommèrent de suivre le mouvement. Je leur dis que je ne pouvais le faire sans ordre, que le poste avait été confié à notre fidélité et que l'honneur nous commandait d'y rester jusqu'au dernier moment - "Eh bien, nous partons tout de même", répondirent-ils. — "Partez si bon vous semble, fis-je, mais je reste à mon poste". Un gendarme sortit des rangs : - "non, mon lieutenant, s'écria-t-il, vous êtes un brave; vous ne resterez pas seul. Je reste avec vous". Et il mit pied à terre. Un autre l'imita, puis le peloton tout entier ${ }^{1}$."

Pendant toutes les révolutions du XIX ${ }^{\mathrm{e}}$ siècle, les gendarmes figurent parmi les plus fidèles soutiens du pouvoir en place. Après son renversement, ils se mettent au service des anciens insurgés, devenus les nouveaux gouvernants. Ce cycle perpétuel est présenté comme la preuve du légalisme de la gendarmerie. Inscrit dans les règlements, ce principe ne s'enracine pourtant pas facilement dans la pratique d'un corps qui confondra longtemps service de l'État et service du prince. Dans le témoignage du lieutenant La Roche, il n'est pas tant question d'obéissance à la loi que de " fidélité " et d'" honneur " - une distinction significative. Entre les textes officiels et la réalité vécue subsiste une importante marge d'incertitude sur laquelle doivent se concentrer les recherches ${ }^{2}$.

1. Souvenirs d'un officier de gendarmerie sous la Restauration publiés et annotés par le vicomte Aurélien de Courson, Paris, Plon, 1914, p. 188-189.

2. Je remercie Arnaud Houte pour sa contribution à cette conclusion. 


\section{Un principe réglementaire : le légalisme de la gendarmerie}

La loi du 28 germinal an VI (17 avril 1798) précise que la gendarmerie est " une force instituée pour assurer, dans l'intérieur de la République, le maintien de l'ordre et l'exécution des lois. Une surveillance continue et répressive constitue l'essentiel de son service ". La primauté du texte législatif est clairement affirmée au cour de cette définition du métier qui reste citée - et enseignée - jusqu'à nos jours. Elle s'affiche sur les premiers étendards du corps, avec la mention " Discipline et obéissance à la loi ", comme sur les boutons de l'uniforme, frappés de la formule «Force à la loi ». Placée au service de l'État, incarnation de la continuité du pouvoir, la gendarmerie est aussi, dès sa création par la Révolution, l'instrument de la souveraineté nationale, qui remplace désormais l'autorité personnelle du roi. Garante des droits de l'homme et du citoyen, selon l'article 12 de la déclaration du 26 août 1789, elle est étroitement subordonnée à la règle de droit. Soumission à l'autorité légitime et défense de la légalité constituent les bases intangibles de son service ${ }^{3}$.

L'organisation hiérarchique du corps et sa culture militaire de l'obéissance doivent garantir la loyauté de ses membres. Contre les tentations autonomistes susceptibles de naître au sein d'une troupe trop indépendante $^{4}$, les textes réglementaires organisent d'abord une mise sous tutelle de l'institution. Tandis que le commandement est réparti entre plusieurs ministères concurrents, la gendarmerie ne dispose pas d'une véritable direction autonome, à la notable exception de l'état-major général du Premier Empire. Cette situation est reproduite à l'échelon de la brigade, où les hommes sont tenus de répondre aux réquisitions réglementaires des autorités, administratives, judiciaires et militaires, tout en restant responsables de leurs opérations. Au niveau des simples gendarmes, enfin, la prestation solennelle du serment rappelle à chaque recrue la nature de ses obligations. "C'est une date dans la carrière ", explique, au début du Xx" siècle, Ignace Forestier, qui compare la cérémonie à une sorte de " sacre $^{5}$ ». Avant d'entrer officiellement en fonction, l'impétrant est conduit en grand uniforme devant le tribunal de première instance, où il prononce une formule quasiment immuable : « Je jure d'obéir à mes chefs en tout ce qui concerne le service auquel je suis appelé et, dans l'exercice de mes fonctions, de ne faire usage de la force qui m'est confiée que pour le maintien de l'ordre et l'exécution des lois. " Mais une déclaration d'allégeance personnelle au souverain, imposée par l'ordonnance de 1820 (article 32), servait aussi de prologue,

3. Sur la définition officielle des missions de la gendarmerie, voir « Le bras armé de l'État ", dans Luc, Jean-Noël (dir.), Gendarmerie, État et société au XIXe siècle, Paris, Presses de la Sorbonne, 2002, p. 31-61.

4. Cette question, classique en sciences politiques, a fait l'objet d'une mise au point synthétique : MANn, Patrice, "Pouvoir politique et maintien de l'ordre : portée et limites d'un débat ", Revue française de sociologie, 1994, p. 435-455.

5. ForestiER, Ignace-Émile, Gendarme à la Belle Époque. Souvenirs recueillis par le colonel Jacques Forestier, Paris, France-Empire, 1983, p. 63-64. 
jusqu'à sa suppression par la Troisième République : « Je jure et promets de bien et fidèlement servir le roi. Banal sans être pour autant anodin dans la première moitié du XIX siècle $^{6}$ ", cet usage rappelle la confusion persistante des principes de loyauté et de légalisme.

Ainsi inculqué et mis en scène, le légalisme détermine la fidélité au pouvoir en place. C'est particulièrement net dans le cas de l'unité spéciale chargée du maintien de l'ordre à Paris et qui joue, de ce fait, un vrai rôle politique au cours du XIX ${ }^{\mathrm{e}}$ siècle. Dans sa thèse sur la garde républicaine, puis garde de Paris, entre 1848 et 1871, Fabien Cardoni a révélé la rivalité de l'État et la ville pour le contrôle de cette troupe, rarement chargée de missions de surveillance politique, mais sans doute l'une des plus politisées de l'arme ${ }^{7}$. En juillet 1830, la gendarmerie royale de Paris charge les émeutiers en exécution des instructions du maréchal Marmont. En février 1848, son héritière, la garde municipale de Paris, défend, sous les ordres de Bugeaud, le trône de Louis-Philippe. Le 18 mars 1871, des hommes de la garde républicaine couvrent la débandade de l'armée de ligne, place Pigalle, lors de la vaine tentative du gouvernement pour récupérer les canons parisiens. À chaque fois, le gendarme est engagé par ses chefs contre une sédition : il ne fait qu'obéir à ses supérieurs et à l'autorité légitime. " Le soldat meurt au poste où on le place. La faute n'est pas au bras qui frappe, mais à la tête qui ordonne ", tient d'ailleurs à rappeler le Journal de la Gendarmerie, peu après les journées de $1848^{\circ}$. C'est dire que l'historien doit se méfier des jugements anachroniques et des raccourcis téléologiques : comment les gendarmes sauraient-ils qu'ils affrontent des révolutions bientôt couronnées de succès? Comment auraient-ils conscience de s'opposer à une hypothétique " marche de l'histoire "? En d'autres occasions, par exemple en 1827, puis au début de la Monarchie de Juillet ou à la fin du Second Empire, les gendarmes ont contenu des manifestations qui n'ont pas réussi à renverser l'autorité établie et à propos desquelles ne s'est pas posée, ensuite, la question du bien-fondé de leur action, sauf pour les opposants aux régimes respectifs. Du point de vue des principes réglementaires, il n'y a donc pas lieu de s'étonner des résistances opposées par la gendarmerie aux insurgés lors des journées révolutionnaires du XIXe siècle.

Le légalisme qui préside à la défense du pouvoir menacé entraîne ensuite la soumission aux nouvelles autorités. Sans doute l'épuration administrative contribue-t-elle à écarter les gendarmes les plus compromis. Mais si le mécanisme fonctionne à plein entre 1814 et 1816, comme le montre la communication d'Édouard Ébel, la première approche statistique globale du sujet, il est nettement moins utilisé en 1830 et en 1848. Quelques

6. On trouvera quelques éléments de comparaison chez PiBoubes, Jean-Yves, Le serment politique en France, 1789-1870, doctorat, histoire, Paris I, dir. Alain CORBIN, 2003, 497 p.

7. CARDoni, Fabien, La Garde Républicaine, d'une République à l'autre. Un régiment de gendarmes à Paris, 1848-1871, doctorat, histoire, Paris IV, dir. Jean-Noël Luc, 2005, 782 p., à paraître.

8. Cité par LARRIEU, Louis (général), Histoire de la maréchaussée et de la gendarmerie des origines à la Quatrième République, Maisons-Alfort, SHGN-Phénix Éditions, 2002, p. 567. 
mois après la chute de Charles X, plusieurs membres des unités parisiennes engagées contre la Révolution de Juillet rentrent dans les bataillons mobiles qui luttent, en province, contre les partisans du monarque déchu, non sans éveiller la méfiance des patriotes locaux ${ }^{9}$ ! Et la recomposition du corps semble encore plus minime après 1848, à l'exception de la "garde " parisienne, à la fois acteur et victime des bouleversements politiques, qui subit six vagues d'épuration en moins d'un an. En fait, les autorités civiles ne tentent de procéder à des épurations importantes qu'en 1849-1850, puis surtout en 1878-1880. La remarquable continuité de la gendarmerie au cours d'un siècle marqué par de nombreux épisodes révolutionnaires inscrit son histoire, comme auparavant celle de la maréchaussée, dans celle de la construction de l'État, c'est-à-dire dans ce long processus de centralisation administrative, d'encadrement des individus et de monopolisation de la violence légitime qui résiste à toutes les secousses politiques.

\section{Une pratique ambiguë : l'utilisation politique de la gendarmerie}

Dans cette perspective, on peut relier la réglementation du métier de gendarme à cette " révolution de la publicité " qui caractérise, selon Pierre Rosanvallon, le «Léviathan démocratique ". Tenue à une " contrainte de transparence ou au moins de plus grande visibilité ${ }^{10}$ " à partir de l'instauration d'un État de droit, et plus encore avec l'avènement des gouvernements représentatifs, l'administration du XIX ${ }^{\mathrm{e}}$ siècle doit théoriquement rester à l'écart des luttes d'influence et du combat électoral. Imprégnée d'une culture militaire qui l'éloigne peut-être plus facilement des querelles partisanes, la gendarmerie semble pouvoir jouer un rôle pionnier dans cette difficile naissance d'une neutralité de l'État. L'ordonnance du 9 octobre 1820 n'impose-t-elle pas fermement à ses membres le port de l'uniforme dans toutes les circonstances de leur service et l'établissement d'un procès-verbal pour toutes leurs opérations? Répétée à plusieurs reprises, l'injonction de transparence est érigée en dogme du métier par l'article 119 du décret du $1^{\mathrm{er}}$ mars 1854 : " Dans aucun cas, ni directement, ni indirectement, la gendarmerie ne doit recevoir de missions occultes de nature à lui enlever son caractère véritable. Son action s'exerce toujours en tenue militaire, ouvertement et sans manœuvres de nature à porter atteinte à la considération de l'arme".

Mais l'ambiguïté des discours officiels éclate quand on quitte l'esprit des règlements pour entrer dans la lettre des instructions. Le 12 novembre 1849, une circulaire «très confidentielle " du ministre de la Guerre, le

9. LIGNEREUX, Aurélien, Force à la loi? Rébellions à la gendarmerie et autorité de l'État dans la France du premier XIX $X^{e}$ siècle (1800-1859), doctorat, histoire, sous la dir. de Nadine Vivier et de Jean-Noël Luc, Université du Maine, 2006, p. 437, à paraître aux PUR. Ce travail montre par ailleurs qu'il ne suffit pas, pour que la transition s'effectue sans heurts, que les gendarmes s'inclinent devant le fait accompli. Il faut que les populations renoncent également aux représailles contre les représentants locaux du régime déchu.

10. Rosanvallon, Pierre, L'État en France de 1789 à nos jours, Paris, Seuil, 1990, p. 24. 
général Alphonse d'Hauptoul, approuvée par les deux tiers de l'Assemblée législative, invite les officiers et les chefs de brigade à l'informer des progrès de la propagande socialiste dans les campagnes et des moyens de la combattre. Mieux encore, deux mois avant la promulgation du décret de 1854, qui interdit toute mission occulte, le général de la Ruë, président du Comité de la gendarmerie, invite discrètement les chefs de légion à poursuivre leur " correspondance politique " sous double enveloppe! Enfin, l'article 286 du décret de 1854 évoque la " surveillance active et persévérante " des condamnés " qui cherchent à faire de la propagande révolutionnaire ". Sur la confusion réglementaire viennent se greffer des recommandations explicites. À partir de 1859, le général de la Ruë s'efforce ainsi d'instaurer un véritable "système d'espionnage politique ", selon les propres termes d'un chef d'escadron retraité ${ }^{11}$. Et lors des élections de 1863, le ministre de l'Intérieur n'hésite pas à demander aux brigades des rapports quotidiens sur le parcours et les entretiens des candidats de l'opposition! On voit que le Second Empire sait ressusciter des dispositifs de surveillance élaborés par le premier empereur ${ }^{12}$. Ressusciter ou réorganiser? L'étude de Christophe Aubert montre qu'ils n'ont jamais été vraiment abandonnés, même si certains officiers témoignent peu de zèle pour cette activité. Comme les commissaires de police étudiés par John Merriman pour le premier XIX ${ }^{\mathrm{e}}$ siècle, les officiers et les chefs de brigade semblent " tout sauf neutres ${ }^{13}$ ". La gendarmerie reste un précieux instrument de police politique, dont aucun gouvernement n'entend vraiment se priver.

L'arme " a péché par implication politique " entre le Premier Empire et 1870, admet d'ailleurs, en 1993, le lieutenant-colonel Marc Watin-Augouard, avec une indépendance d'esprit qui tranche sur l'historiographie officielle, mais elle trouve ensuite une place légitime " dans l'affirmation de l'État de droit ${ }^{14}$ ". Idée partagée par des juristes comme Marceau Long, vice-président du Conseil d'État, pour lequel l'avènement de la Troisième République permet à la gendarmerie d'incarner " l'ordre respectueux des libertés ». Et au début des années 1990 encore, le politologue François Dieu voit dans cette étape le début de "l lâge d'or de la gendarmerie ", puisque l'idéologie républicaine affirme le caractère « exclusivement légaliste » de son activité policière ${ }^{15}$. Si de nombreux indices témoignent de cette conversion, les

11. Voir notamment Houte, Arnaud-Dominique, Le métier de gendarme national au XIX siècle. Pratiques professionnelles, esprit de corps et insertion sociale de la Monarchie de Juillet à la Grande Guerre, doctorat, histoire, sous la dir. de Jean-Noël Luc et Jean-Marc Berlière, Paris IV, 2006, p. 247-258, à paraître aux PUR.

12. LignereuX, Aurélien, Gendarmes et policiers dans la France de Napoléon. Le duel Moncey-Fouché, Maisons-Alfort, SHGN, 2002, 275 p.

13. MerRiman, John M., Police Stories. Building the French State, 1815-1851, Oxford, Oxford University Press, 2006, p. 9.

14. PicARD, Étienne, RichaRdot, Michel, Watin-Augouard, Marc, " Gendarmerie-police, une dualité en débat ", Cahiers de la sécurité intérieure, n 11, novembre 1992-janvier 1993, p. 196.

15. LoNG, Marceau, «La gendarmerie et l'État de droit ", Colloque international du bicentenaire de la gendarmerie in Revue de la gendarmerie, $\mathrm{n}^{\circ} 167,3^{\mathrm{e}}$ et $4^{\mathrm{e}}$ trimestre 1991, p. 7; 
travaux les plus récents montrent qu'elle commence seulement à la fin des années 1870. Il faut effectivement attendre la chute de "l'ordre moral " pour que plusieurs instructions proscrivent formellement l'immixtion de la gendarmerie dans "des questions qui touchent à la politique ", tandis que le serment professionnel s'émancipe des déclarations de fidélité au chef de l'État. Et les journaux corporatifs veillent attentivement au respect de ces nouveaux principes. Sans doute des dérapages se produisent-ils, notamment quand un officier décide de protéger le candidat de son choix lors d'une campagne électorale. Mais la thèse d'Arnaud-Dominique Houte, déjà citée, sur la culture professionnelle de la gendarmerie entre les années 1830 et la Grande Guerre révèle bien la rupture provoquée par la victoire des républicains. Désormais, le discours identitaire de l'arme valorise clairement la neutralité absolue de ses membres. Parfaitement neutre pendant la crise boulangiste ou lors de l'affaire Dreyfus, l'institution se prête aussi bien à la répression des grèves qu'à la lutte contre les congrégations. Le Journal de la Gendarmerie s'en réjouit d'ailleurs, en mars 1905 : " Notre arme a été attaquée tout à la fois par la droite et par la gauche, voilà la preuve de sa neutralité ${ }^{16}$."

Toujours dociles, mais beaucoup plus méfiants à l'égard des directives officieuses, les gendarmes apprennent à tracer une ligne de démarcation entre la police administrative et la police politique. Il est vrai qu'il n'est pas toujours aisé de distinguer la collecte du « bon renseignement " et l'espionnage partisan. Encore aujourd'hui, la circulaire du 8 novembre 1985 rappelle que " tous les renseignements, de quelque nature qu'ils soient, intéressent la gendarmerie, à l'exclusion toutefois de ceux qui se rapportent aux questions politiques ". Si cette définition a le mérite de la concision, elle postule l'existence d'une frontière qu'il n'est pas toujours facile d'identifier dans la pratique quotidienne du renseignement. Qu'est-ce qui relève de la " politique "? La question est encore plus complexe au XIX ${ }^{\mathrm{e}}$ siècle. Comme l'a montré Pierre Karila-Cohen, les monarchies censitaires accordent la plus grande attention à l'" esprit public ${ }^{17}$ ». Les rapports amassés brassent toutes sortes de détails, depuis le prix des céréales ou l'évolution du chômage des ouvriers jusqu'aux rumeurs de guerre ou aux activités de l'opposition. Chevilles ouvrières de la collecte de l'information, les gendarmes réunissent les données brutes. On ne leur en demande généralement pas plus. D'une part, car leur éthique militaire les incite à une certaine réserve; d'autre part, car les autorités ne sont pas curieuses de lire, selon l'expression d'un

François Dieu, Gendarmerie et modernité : étude de la spécificité gendarmique aujourd'hui, Paris, Montchrestien, 1993, p. 138.

16. Cité par Arnaud-Dominique Houte, op. cit., p. 657.

17. KarILA-CoHEn, Pierre, "L'état des esprits ": l'administration et l'observation de l'opinion départementale sous la monarchie constitutionnelle (1814-1848), doctorat, histoire, sous la dir. d'Alain Corbin, Paris I, 2003, 691 p. Voir aussi " Une " bonne surveillance ": la gendarmerie et la collecte du renseignement politique en province sous la monarchie censitaire ", dans Luc, Jean-Noël (dir.), Gendarmerie, État et société au XIX siècle, op. cit., p. $225-236$ 
préfet du Second Empire, «l'appréciation peu convenable d'un brigadier souvent peu intelligent et presque toujours peu éclairé ${ }^{18}$ ". Les rapports de gendarmerie rassemblent des renseignements plutôt décousus et souvent anodins : la part conférée à l'information administrative y dépasse largement celle de l'espionnage au sens strict. Mais en transmettant toutes les informations qu'elles recueillent, les brigades n'en sont pas moins un rouage essentiel du dispositif de police politique.

Les frontières sont aussi floues en matière de maintien de l'ordre. Où s'arrête le strict exercice du maintien de l'ordre? Où commence l'atteinte aux libertés publiques - Christophe Aubert a bien posé ici la question - et par exemple à travers l'intimidation des opposants? La confusion est aussi extrême dans le cas des barricades du XIX ${ }^{\mathrm{e}}$ siècle : les gendarmes se battent autant pour rétablir l'ordre sur la voie publique que pour protéger le pouvoir en place. D'autres types d'interventions soulèvent les mêmes interrogations. Dans quelle catégorie faut-il classer, par exemple, les arrestations des auteurs de propos séditieux, encore poursuivis dans la première moitié du XIX ${ }^{e}$ siècle? Que faire de ces "dizaines de petits coups d'État locaux " signalés par Maurice Agulhon sous la Seconde République ${ }^{19}$ ? Comment interpréter les tracasseries dont sont victimes les concurrents des candidats officiels lors des élections du Second Empire? Ces questions rebondissent sous la III ${ }^{e}$ République, lorsque la gendarmerie surveille de très près les parlementaires socialistes qui viennent soutenir un mouvement de grève. On le comprend : la lecture des règlements ne peut pas être dissociée d'une analyse détaillée des pratiques et de leurs ambiguïtés.

\section{Les questions de l'historien}

Aujourd'hui comme hier, les commentateurs aiment souligner le légalisme foncier de la gendarmerie. À juste titre, puisque ce principe sans cesse rappelé aide l'historien à mieux comprendre l'attitude de cette institution pendant les périodes de crise. Mais lorsqu'elle devient exclusive, cette explication entrave la recherche. Elle fait oublier que ce légalisme s'incarne, à chaque étape de l'histoire politique du XIX ${ }^{\mathrm{e}}$ siècle, au service d'un pouvoir particulier et dans des affrontements avec les adversaires de ce pouvoir. Ce constate inspire au moins trois directions de recherche.

Qu'en est-il, d'abord, du rôle exact de la gendarmerie au cours de l'ensemble des périodes de crise et de transition politique? De la Révolution aux affrontements religieux ou sociaux de la Belle Époque, l'historien du $\mathrm{XIX}^{\mathrm{e}}$ siècle n'a que l'embarras du choix. Que font les gendarmes entre la Première Restauration et la fin des Cent Jours? Que se passe-t-il à Paris, mais aussi en province, lors des journées de juillet 1830 ou de février 1848 (ces dernières ont déjà été bien étudiées pour la capitale dans la thèse

18. Cité par Arnaud-Dominique Houte, op. cit., p. 254-255.

19. Agulhon, Maurice, 1848 ou l'apprentissage de la République, Paris, Seuil, 1973, p. 179. 
de Fabien Cardoni) ${ }^{20}$ ? Après les excellents travaux déjà réalisés, n’y a-t-il plus rien à découvrir à propos de la participation de l'arme à la forfaiture de décembre $1851^{21}$ ou à la répression de la Commune ${ }^{22}$ ? Comment faut-il interpréter l'incorporation d'officiers de gendarmerie dans les cours prévôtales hâtivement constituées en 1871 ? Si des vues d'ensemble ont été tracées pour certaines de ces questions, plusieurs enquêtes approfondies restent nécessaires, par exemple pour étendre les investigations, à la suite de la communication d'Aurélien Lignereux, à l'action de la gendarmerie lors des sorties de crise. Comment les brigades se soumettent-elles concrètement au nouveau pouvoir? Quand reprennent-elles leurs missions habituelles? Comment sont-elles considérées par les nouveaux dirigeants et par les populations?

Répondre à ces diverses questions exige d'observer les répercussions des crises sur l'image du corps et notamment sur ses relations avec ses administrés. L'intervention parfois zélée des gendarmes dans la répression politique les coupe toujours d'une partie de la population et noircit leur image dans la mémoire collective. Après la chute de Charles X, que sa fidèle gendarmerie d'élite accompagne sur la route de l'exil, des acteurs des Trois Glorieuses dénoncent les tirs sans sommation des unités parisiennes, qui sont aussitôt dissoutes. Sous le Second Empire, le souvenir des exactions de 1852 et de 1858 poursuit le bicorne, en particulier dans les régions méridionales, où la qualité de proscrit de 1851 reste un titre de noblesse, tandis que l'opposition républicaine accuse l'arme de se comporter en vulgaire milice politique. Longtemps après la Commune, des républicains reprochent aux gendarmes l'exécution des prisonniers, le meurtre de Gustave Flourens, ancien professeur au Collège de France et général du camp insurgé, et les méthodes très expéditives des cours prévôtales.

Comme toutes les situations exceptionnelles abondamment documentées, les crises politiques ouvrent une nouvelle fenêtre sur le fonctionnement de la gendarmerie du $\mathrm{XIX}^{\mathrm{e}}$ siècle. Les communications rassemblées ici le démontrent sur des registres différents. Grâce à la communication d'Édouard Ébel, on connaît bien, désormais, le mécanisme et l'ampleur des épurations de 1814-1816, mais le travail est à poursuivre pour la suite du siècle. Même si l'on sait que les autres renouvellements sont moins massifs, chaque épuration reste une formidable fabrique d'indices pour l'historien. Et d'autant plus que la menace, parfois, ne vise pas seulement le personnel, mais aussi l'institution elle-même, comme le montre Jean-François Tanguy en étudiant les projets de réforme des années 1870. Les répercussions plus lointaines des expériences de crise constituent un autre champ de

20. CARDoni, Fabien, La Garde Républicaine..., op. cit., p. 86-170.

21. La meilleure synthèse reste celle de MARGADANT, Ted, French Peasants in Revolt. The Insurrection of 1851, Princeton, Princeton University Press, 1979, 379 p. Le rôle de la gendarmerie est précisé dans les récentes thèses de Fabien CARDONI (op. cit., p. 217-228), d'Arnaud-Dominique, HouTE (op. cit., p. 150-158) et d'Aurélien LiGNEREuX (op. cit., p. 578-588)

22. Doessant Benoît, La Gendarmerie et la Commune de Paris, maîtrise, histoire, dir. Jean-Noël Luc, Paris IV, 2000, 168 p. 
recherche : à l'aide d'un vaste corpus inédit, Jean-François Tanguy décrit les adaptations des pratiques et des discours dans le contexte de la remise en cause des années 1870. À un niveau plus général, Arnaud-Dominique Houte rappelle les liens entre la crainte de l'épuration et un repli prudent sur la dépolitisation qui s'enracine dans l'imaginaire du métier tout en déterminant les positionnements sociaux des gendarmes de la seconde moitié du XIX ${ }^{\mathrm{e}}$ siècle. L'enquête ne pourra pas se poursuivre sans aborder la question des préférences politiques des officiers à la lumière des remarques d'Olivier Forcade sur "l'histoire politique plurielle " des cadres de l'armée ${ }^{23}$. Peut-être éclairera-t-on ainsi les formes et les limites éventuelles de la dépolitisation républicaine de la gendarmerie ou les décalages entre la troupe et les officiers.

L'analyse de l'évolution des tactiques du maintien de l'ordre par Fabien Cardoni incite à étudier aussi les répercussions des troubles liés aux transitions politiques sur la gestion de l'ordre public urbain. La responsabilité des charges, l'usage des armes, la pratique ou non des sommations, sont autant de zones d'ombre et de sujets de débats ${ }^{24}$ pour lesquels on attend plusieurs études qui permettront de préciser - et peut-être de nuancer? - le légalisme de la gendarmerie dans des contextes particuliers. Et la thèse d'Aurélien Lignereux élargir la perspective pour le premier XIX ${ }^{\mathrm{e}}$ siècle en offrant la possibilité de comparer les réactions des gendarmes pendant les journées insurrectionnelles et leurs répliques aux rébellions collectives régulières ${ }^{25}$. Les périodes de troubles permettent également d'étudier les relations entre la gendarmerie et les autres forces de l'ordre - garde nationale, police, armée de ligne ou autres unités nées de l'insurrection. Elles peuvent aussi éclairer le fonctionnement concret d'une institution définie par l'association d'une "centralisation contrariée ", d'une "structure déconcentrée " et d'une " gestion collégiale ", parfois conflictuelle, avec les autorités civiles ${ }^{26}$. À quel principe, à quelle autorité, doit s'en remettre, en temps de crise, le chef de brigade isolé dans son canton? Quels sont, selon les contextes et les individus, les effets du principe hiérarchique, de l'acculturation gendarmique, mais aussi des préoccupations personnelles, comme le souci de conserver son emploi? Autant de questions qui permettront de prolonger les analyses d'Arnaud-Dominique Houte sur la pro-

23. FORCADE, Olivier, " L'histoire politique des armées et des militaires dans la France républicaine (1871-1996) : essai d'historiographie ", Jean Jaurès. Cahiers trimestriels, $\mathrm{n}^{\circ} 142$, octobre-décembre 1996, p. 17. On pourra également se reporter aux analyses pionnières de EmsLEy, Clive, Gendarmes and the State in Nineteenth Century Europe, Oxford, Oxford University Press, 1999, p. 258-259.

24. Le général Larrieu signale également la défaillance des autorités civiles, en l'occurrence des commissaires de police, qui se sont abstenus de venir faire les sommations d'usage sur le terrain; L. LARRIEU, op. cit., p. 557-559.

25. La thèse déjà citée d'Aurélien LIGNEREUX permet ainsi de suivre, de 1800 à 1859, l'évolution de l'usage effectif des armes au cours de quelque 3706 rébellions collectives reconstituées dans le détail de leur déroulement.

26. CaRTAYRAdE, Cyril, La gendarmerie nationale du Centre-Est de la France, 1791-1854, DEA, histoire, sous la dir. d'Olivier Faure, Lyon III, p. 36-75. 
fessionnalisation du métier de gendarme et sur l'émergence d'une éthique de responsabilité ${ }^{27}$. Autant de questions, surtout, qui incitent à élargir les investigations aux transitions du Xxe siècle, à l'avènement puis à la chute de Vichy ${ }^{28}$ ou aux crises de la décolonisation.

\section{RÉSUMÉ}

Pendant toutes les révolutions du $\mathrm{XIX}^{\mathrm{e}}$ siècle, les gendarmes figurent parmi les plus fidèles soutiens du pouvoir en place. Après son renversement, ils se mettent au service des anciens insurgés, devenus les nouveaux gouvernants. Ce cycle perpétuel est présenté comme la preuve du légalisme de la gendarmerie. Inscrit dans les règlements, ce principe ne s'enracine pourtant pas facilement dans la pratique d'un corps qui confondra longtemps service de l'État et service du prince. Entre les textes officiels et la réalité - notamment l'utilisation de la gendarmerie comme police politique - subsistent des contradictions, des ambiguïtés, des zones d'ombre, sur lesquelles doivent se concentrer les recherches.

\section{ABSTRACT}

In every revolution of the nineteenth century, Gendarmes are always one of the staunchest supports of the incumbent regime. After it is overthrown, they serve the formerly insurgents turned new rulers. This perpetual cycle is shown as proof of Gendarmerie's legalism. Recorded in its rulebooks, this principle does not easily for a long time, mistook service of the State with service of the Prince. Between texts and reality, notably regarding the use of the Gendarmerie as a political police, there remains some contradictions, ambiguities, and unknowns that new workings should concentrate on.

27. HouTe, Arnaud-Dominique, op. cit., notamment p. 396-400, 415-417 et 751-759.

28. Des questions notamment étudiées dans les thèses en préparation d'Olivier BUCHBINDER, La Gendarmerie et le rétablissement de la légalité républicaine de 1944 à 1947 (Paris IV); CHEVET, Emmanuel, Gendarmerie et maquis en France sous l'Occupation (Dijon); CAmpION, Jonas, Le Rétablissement de la légalité policière après la Seconde Guerre mondiale : la gendarmerie belge, la gendarmerie française et la Koninklijke maréchaussée hollandaise (université catholique de Louvain et Paris IV) et NATIVITÉ, Jean-François, La Gendarmerie sous l'Occupation dans les départements pyrénéens (Montpellier III), ainsi que dans les actes de la journée d'étude organisée, en octobre 2006, par la Société Nationale Histoire et Patrimoine de la Gendarmerie et l'université Paris IV : La gendarmerie et les gendarmes de 1939 à 1945. Entre l'État et la nation?, à paraître, en 2007, dans le nº 2 de Force publique; voir aussi BERGÈRE, Marc, " Pratiques et bilan de l'épuration administrative des officiers de gendarmerie à la Libération ", dans Luc, Jean-Noël (dir.), Gendarmerie et gendarmes du XX $x^{e}$ siècle, Paris, Publications de la Sorbonne, à paraître. 